\title{
Smart dressings for wound monitoring and treatment: new-type skin bioelectronic systems applicated in wound repair
}

\author{
Yu-Fan Zhong ${ }^{1}$, Yong Wang ${ }^{1}$, Yan-Yan Hu${ }^{1}$, Xiao-Feng Wang ${ }^{1}$, Fa-Wei Xu ${ }^{1}$, Yan-Qi Liu ${ }^{1}$, \\ Qing-Qing Fang ${ }^{1}$, Wan-Yi Zhao ${ }^{1}$, Ting-Wen Kuo ${ }^{1}$, Ya-Nan Xue ${ }^{1}$, and Wei-Qiang Tan ${ }^{1}$ \\ ${ }^{1}$ Zhejiang University School of Medicine Sir Run Run Shaw Hospital
}

October 20, 2021

\begin{abstract}
Wound management, especially for chronic wounds, has emerged as a major healthcare challenge, which brings great pain and other negative impacts to patients, and accounts for significant portions of health care budgets. The common practice of covering wounds with dressings, owing to the lack of information about wound healing underneath, cannot provide insights into the status of the wound. Changing the dressings to inspect wounds not only disturbs normal healing process of wounds, but also causes pain to affected individuals. Therefore, it is necessary to rely on parameters in the wound microenvironment such as temperature, $\mathrm{pH}$, moisture level, and etc., by continuously monitoring, to indicate the wound status and healing stages. Moreover, intelligent on-demand treatment systems also can be integrated into the smart dressing, called new-type skin bioelectronic systems to be applicated in wound repair. Besides monitoring, these kinds of skin bioelectronic systems can offer effective treatments automatedly when the wound deteriorates. It makes timely treatment possible and avoids delayed treatment and uncovering the dressing to give medicine. In this review, wound healing-related indexes in the wound microenvironment are discussed, applications of various smart dressings with functions of monitoring or/and treatment are summarized.
\end{abstract}

Smart dressings for wound monitoring and treatment: new-type skin bioelectronic systems applicated in wound repair

Yu-Fan Zhong ${ }^{1}$, Yong Wang ${ }^{1}$, Yan-Yan $\mathrm{Hu}^{1}$, Xiao-Feng Wang ${ }^{1}$, Fa-Wei Xu${ }^{1}$, Yan-Qi Liu ${ }^{1}$, Qing-Qing Fang ${ }^{1}$, Wan-Yi Zhao ${ }^{1}$, Ting-Wen Kuo ${ }^{1}$, Ya-Nan Xue ${ }^{1}$, Wei-Qiang Tan ${ }^{1 *}$

${ }^{1}$ Department of Plastic Surgery, Sir Run Run Shaw Hospital, School of Medicine, Zhejiang University, Hangzhou, 310016, Zhejiang Province, P.R. China.

${ }^{*}$ Correspondence:

Wei-Qiang Tan

Department of Plastic Surgery, Sir Run Run Shaw Hospital, School of Medicine, Zhejiang University, Hangzhou, 310016, Zhejiang Province, P.R. China

E-mail address:tanweixxxx@zju.edu.cn

\section{ABSTRACT}

Wound management, especially for chronic wounds, has emerged as a major healthcare challenge, which brings great pain and other negative impacts to patients, and accounts for significant portions of health care budgets. The common practice of covering wounds with dressings, owing to the lack of information about wound healing underneath, cannot provide insights into the status of the wound. Changing the dressings to inspect wounds not only disturbs normal healing process of wounds, but also causes pain to affected individuals. Therefore, it is necessary to rely on continuously monitoring parameters in the wound microenvironment 
such as temperature, $\mathrm{pH}$, moisture level and etc., by sensors to indicate the wound status and healing stages. Moreover, intelligent on-demand treatment systems also can be integrated into the smart dressing, called new-type skin bioelectronic systems to be applicated in wound repair. Besides monitoring, these kinds of skin bioelectronic systems can offer effective treatments automatedly when the wound deteriorates. It makes timely treatment possible and avoids delayed treatment and uncovering the dressing to give medicine. In this review, wound healing-related indexes in the wound microenvironment are discussed, applications of various smart dressings with functions of monitoring or/and treatment are summarized.

\section{Keywords}

Smart dressings; Wound healing; Monitoring; On-demand treatment; Wound microenvironment; Sensors

\section{Introduction}

Skin, which covers the body surface and is in direct contact with the external environment, is the largest organ, accounting for $16 \%$ of the total body weight, with a total area of about 1.5-2 square meters. It can not only resist the invasion of external microorganisms, protect our body from the harm of external mechanical forces, thermal radiation and chemical stimulation, but also feel changes of external environment then adjusts the balance of water and salt in our body, regulate body temperature and so on. What's more, skin also plays a very important role in maintaining the appearance of human body. However, it is inevitably subjected to a variety of damage due to long-term and large-area skin exposure to the external environment. Skin wound caused by severe trauma, surgical injury or chronic diseases (cardiovascular disease, diabetes, etc.), often manifested as direct defect, unhealed inflammation and ulcer formation, is one of the most serious diseases in today's society. Wound repair is a complex, dynamic, and multi-factorial physiological process which involves proliferation, differentiation, migration, and matrix synthesis and deposition of a variety of cells, and is regulated by various growth factors and biological signals(Tottoli et al., 2020). The process of wound healing can be divided into four overlapping phases: hemostasis, inflammation, proliferation and finally remodeling(Sun et al., 2014). Once a certain step in the healing process is in disorder, wound healing will be blocked or even not healed for a long time. At present, those the course of healing is more than 3 months can be defined as chronic wounds, whose condition is often complicated due to the long course of healing and complications accompanied. As population ages in our country, the incidence of chronic wounds is increasing year by year. Tens of millions of people around the world need to be treated for skin defects every year, and millions of them need the treatment of skin grafting in China alone.

Autologous skin transplantation is the standard treatment for skin defects, but is limited by insufficient donor area. In addition, a second operation usually is required after the autologous skin transplantation, which easily triggers complications such as infection and brings great pain and surgical risks to patients. Therefore, dressings, artificial skin substitutes, wound intervention agents (drugs, growth factors, peptides, etc)(Moura et al., 2013) and other methods are more widely used to treat skin defects. Compared with traditional gauze dressings, new types of wound dressings (such as membrane, sponge, hydrogel)(Broussard and Powers, 2013; Simões et al., 2018) and artificial skin substitutes can seal and protect the wound in time, but they cannot provide targeted and tailored interventions according to different types of wounds, which makes it difficult to achieve satisfactory therapeutic effects. In addition, dressing change is another difficulty in clinical practice, as the wound healing process is often in a black-box state, making when to change the dressing a real problem that need to be taken into consideration. In most cases, the medical staff can only judge the wound healing by their personal experience. For example, changing the dressing too early may cause unnecessary pain to patients and may also interfere with the normal wound healing process. And under the condition of too-late dressing change, complications, if occur, are hard to be found and treated in time, which exacerbates the unhealing state of wounds. Therefore, an intelligent dressing which can not only speed up wound healing, but also offer real-time information about the status of wound during healing or even can give on-demand treatment will be an effective means of treating skin defects.

In short, functions of an ideal smart dressings can be concluded into three points. The first and most basic role of any dressing should be tissue and functional replacement. Wound dressings play a vital role in 
promoting wound healing, replacing the skin to function as protection and perception. It also can fit the skin surface very well and is easily adapted to most mechanical stretching. Secondly, smart dressings would better monitor and then offer information about the status of wound healing in real time, which helps clinicians make appropriate decisions, changing the dressing, take medication or etc. And this function cannot be realized without sensors integrated in dressings which can automatically and continuously monitor on or more indicators in the wound microenvironment, and micro-electronic devices that can process and transmit data to the terminal. In 2015, for the first time, some scholars divided the microenvironment affecting skin wound healing into two parts(Kruse et al., 2015), that is, the "external microenvironment" of the wound, directly adjacent to the wound surface; and the "internal microenvironment", the peripheral compartment beneath the wound surface and occupied by various cells and extracellular matrix. Among them, indexes that can reflect the information of wound mainly include temperature, $\mathrm{pH}$, hydration or moisture level, protease and so on. Intact skin is naturally acidic and that helps protect the organism from bacteria and fungi. The wound bed may become more acidic or more alkaline depending on the pathophysiology of the wound, the former usually existing in acute wounds and the latter being associated with a non-healing wound or infected(Power et al., 2017). Monitoring the $\mathrm{pH}$ of a wound may help appropriate measures be taken and promote wound healing. Temperature has long been recognized as an important factor in wound healing, and to some extent it can predict the status of a wound. "Red and hot-touching" were used as an index of poor wound healing in ancient Chinese medicine, while "redness, swelling, heat and pain" were also used as the four classic signs of wound infection in western medicine. Although sometimes elevated temperature is a transient manifestation of wound healing, an abnormal or persistent increase in wound temperature, especially a sudden increase in chronic wounds, is considered to be a sign of wound infection(Woo and Sibbald, 2009; Fierheller and Sibbald, 2010). In addition, hydration is also a vital factor in the microenvironment of wounds. A moist or wet environment improves the speed and quality of wound healing(Lee et al., 2019), and scar formation is reduced in wet wounds compared with dry wounds(Reish et al., 2009). Besides, other indexes such as uric acid, protease, nitric oxide (NO) and etc. are also meaningful factors that can directly or indirectly change the wound microenvironment and ultimately affect the process of wound healing. Therefore, it is necessary and of great importance to detect those indicators of wound healing. The sensor (one or more) on the dressing can collect the data of indicators related to wound healing in real time, and display the measured data on the intelligent terminal through data processing, conversion and wireless transmission, so as to help the medical staff understand the status of wound healing and judge the healing process.

The last function of an ideal smart dressings should be on-demand treatment for wounds. It can not only monitor specific physiological parameters in real time, but also combine the intervention system. This system can intelligently and automatically give corresponding measures according to the actual state of the wound which is speculated from the data detected by sensors, realizing the on-demand treatment. Therefore, this paper reviews smart dressings in the research, most of which can monitor specific indicators of the wound microenvironment, and some dressings also have the role of on-demand treatment of wounds. The following will introduce the development of smart dressings with wound monitoring functions and on-demand treatment functions.

\section{Real-time monitoring}

Wound healing is a complex and delicate biological process involving many factors such as cells, extracellular matrix and cytokines. Research have identified some monitoring parameters that are meaningful for wound healing, such as $\mathrm{pH}$, temperature, humidity, microbial load, protease, etc. These indexes are critical and can be identified as potential indicators for the management and treatment of chronic or non-healing wounds, as they provide information of wound healing progression when monitored and analyzed in real time.

Temperature monitoring

Wound temperature, mainly determined by the ambient temperature and blood flow, has long been recognized as an important factor in wound healing. Existing studies reported two clinically significant aspects of wound temperature: the absolute temperature of the wound and the wound temperature relative to the periwound intact area. The temperature in an acute spontaneously-healing wound usually increases due to local 
vasodilation mediated by vasoactive molecules, allowing the body to deliver more oxygen and nutrients to the injured area(Siah et al., 2019). So, it is warmer than $37^{\circ} \mathrm{C}$ but not significantly warmer than the adjacent skin(Martínez-Jiménez et al., 2013). While in most chronic wounds that tend to occur in the lower extremity, the temperature is approximately 5 degrees Celsius lower than core temperature due to impaired blood supply and oxygenation(Fierheller and Sibbald, 2010). And a sudden increase of temperature is thought to be an early sign of infection before obvious change to the appearance of wounds are observed(Nakagami et al., 2010). Therefore, monitoring temperature continually contributes to judging the wound state and healing process.

Compared with palpation, contact electronic thermometer and infrared hand-held non-contact thermometer(Armstrong et al., 2006) or thermography(Hazenberg et al., 2014; Dini et al., 2015; Siah and Childs, 2015) has been widely used in clinical physical examination in order to judge skin temperature. The contact thermometer is not suitable for the practical application environment of wound bandaging considering its required contact position and contact time. And although the infrared thermometer has the characteristic of reading the value immediately, its application is also limited by the barrier effect of wound dressings. Therefore, miniature temperature sensors emerged and have been developed as they avoid the disadvantages above and can be implanted in wound dressings to collect wound temperature information in real time.

In recent years, epidermal electronics, whose sensors for temperature, $\mathrm{pH}$ and other biomarkers, could be used as diagnostic tools in healing process, are being developed(Dargaville et al., 2013). For example, one skin-like electronics platform was fabricated, which can laminate at wounds reversibly and show real-time temperature distribution by measuring the electrical resistance(Lee et al., 2015). There is also a similar epidermal electronic system (EES) as shown in Figure 1whose sensors are microscale, metal-traces in fractal layouts on soft and elastomeric membranes(Hattori et al., 2014). The copper electrode is designed into an ultra-thin wire-like serpentine structure, enabling conformal lamination to the skin surface. This system is not only capable of measuring and mapping skin temperature, recording thermal conductivity of skin tissue, but delivering precise levels of heating to promote wound healing. What's more, Lou et al. (2020) reported a flexible wound healing system (FWHS) as shown in Figure $\mathbf{2}$ for temperature monitoring and infection early warning. This system has a double-layer structure: temperature sensing layer and collagen-chitosan dermal equivalent layer which is pro-regenerating for wound healing. In a wound, temperatures can be continuously detected by the sensor, then data is processed by the circuit and transferred wirelessly to the customized app. If abnormal temperature was detected, the system would automatically alarm, warning the infection. In summary, this FWHS, integrating the properties of pro-regeneration and real-time wound temperature monitoring, can overcome the "Black-Box" status of wound healing so as not to change the dressing too often. Though remaining in the state of proof of concept, this FWHS is expected to be used clinically in future.

Radiofrequency Indentification (RFID) technology has also been used in wireless temperature measurement. Milici et al. (2014) proposed the first example of RFID passive temperature epidermal sensor experimented in real applications. The mechanism behind it is based onto a new family of RFID microchips equipped with an integrated temperature sensor. The sampled temperature can be acquired in a digital form up to a distance of 1 metre. As RFID can conjugate sensing and communication capabilities with no need of local power supply, it overcomes the problem of including a local energy source in dressings(Occhiuzzi et al., 2013; Amendola et al., 2014). With either semiconducting or metallic character, carbon nanotubes (CNT) show an electric resistance strictly dependent on temperature. Matzeua et al. (2011) chose it as sensing film measured by an RFID tag to continuously monitor temperature, and is believed to predict initiation of pressure ulcers and upcoming inflammation in the near future.

To sum up, temperature, as an important factor in wound healing, is indicative of wound healing status. And these micro-sensors integrated in wound dressings make real-time monitoring of wound temperature possible, further help clinicians have a good understanding of wound status and then take proper measures if needed.

pH Monitoring 
Normally the $\mathrm{pH}$ of healthy skin is acidic in the range of $\mathrm{pH} 4-6$, due to the secretion of sebaceous glands and other factors(Maver et al., 2019). The acidic microenvironment functions as a barrier against the colonization and invasion of bacteria(Jones et al., 2015). But This barrier will be disturbed when skin integrity is damaged. The $\mathrm{pH}$ change of wounds follows a relatively simple pathway in acute wounds, experiencing transient physiological acidosis before turning into alkaline, then through a more basic granulation step before returning to baseline during re-epithelization(Lindley et al., 2016). The more acidic environment is thought to decrease the risk of infection and can facilitate granulation tissue formation. Vitro studies have demonstrated that an acidic environment helps the activities of keratinocytes and fibroblasts(Percival et al., 2014). However,the circumstance in chronic wounds is more complex: blocked in the inflammatory stage for a long time and failing to heal with $\mathrm{pH}$ oscillating between 7 and 8(Lindley et al., 2016). In such alkaline environment, chronic wounds are easily infected with invasive bacteria, which impeding healing(Bjarnsholt, 2013). Moreover, proteases secreted by the host or bacteria also exhibit dramatically greater activity in alkaline environment, facilitating proteolysis and impair healing(Menke et al. 2007). Wound $\mathrm{pH}$ can also be used to predict healing outcomes and guide treatment. Sharpe et al. (2013) observed that exudate from burn wounds that failed to heal were alkaline, while that of burns healed were acidic. Therefore, the variations of $\mathrm{pH}$ value can act as a significant reference for predicting wound infection and non-healing(Shi et al., 2011).

One of the simplest and most cost-effective method for measuring $\mathrm{pH}$ is using indicator dyes which absorb different wavelengths of visible light depending on the $\mathrm{pH}$. For example, $\mathrm{pH}$-sensitive polymeric dyes were fabricated by grafting phenol red (PR) and rosolic acid (RA) onto chitosan (CS), then it was blended with $\mathrm{CS}$ to make colorimetric $\mathrm{pH}$-sensing films whose color could change in the $\mathrm{pH}$ range 4-10(Chalitangkoon and Monvisade, 2021). In the study of Thet et al. (2016), $\mathrm{pH}$ variation was reflected by fluorescence intensity as the amount of fluorescent dye released from nanocapsules varied according to the changes of $\mathrm{pH}$. Also, Pyranine-modified nanophase-separated amphiphilic polymer conetworks (APCNs) are reported as fluorescent ratiometric $\mathrm{pH}$ sensors(Ulrich et al., 2019). The ratiometric $\mathrm{pH}$ detection and high photostability make it a prime candidate for $\mathrm{pH}$-based chronic wound monitoring. Some studies also combined $\mathrm{pH}$ sensitive dyes with hydrogel to form $\mathrm{pH}$ sensitive hydrogel which then was cast on smart wireless dressings for longterm and remote pH monitoring (Tamayol et al., 2016; Jankowska et al., 2017; Kassal et al., 2017; Liu et al., 2017). Asmaa et al. reported a smart herapeutic pH-sensitive wound dressing based on chitosanbased hydrogel which encapsulates red cabbage extract (RCE)(Arafa et al., 2021). RCE consists of bioactive compounds that are antioxidant, anti-inflammatory, bactericidal, and is also a natural $\mathrm{pH}$-sensitive indicator which shows color changes from green to red when $\mathrm{pH}$ varies from 9 to 4 . While the mechanism of the hydrogel-dependent pH monitor reported by Schneider et al. (2007) is different. It relies on the inductance change of the pair of coils which are sandwiched with a $\mathrm{pH}$-sensitive hydrogel. The distance separating the two planar coils changes when the hydrogel swells and dewells with $\mathrm{pH}$, which causes the change of inductance and the according $\mathrm{pH}$ value are continuously tracked. But the problem is that the deformation of the hydrogel is easily affected by other factors. Some monitoring devices also utilize the mechanism of voltammetric analysis which realized by redox indicators to provide the analytical signal. The peak positions of redox indicators are $\mathrm{pH}$ sensitive, so $\mathrm{pH}$ can be determined by voltammetric analysis of shift in positions. But one study chose uric acid as a redox biomarker(Phair et al., 2011). Uric acid is endogenous to the wound fluid itself, which avoids the ambiguities that may arise from colorimetric analysis, especially given the complexity of wounds fluid ranging from the deep red to the near colorless clarity. Voltage response of metal/metal oxide electrode have been used to detected $\mathrm{pH}$ values due to their low manufacture costs, compatibility with miniaturization processes, high sensitivity, and abundancy(Zeng et al., 2012). For example, one Metal/metal oxide-based $\mathrm{pH}$ sensor, where WO3 nanoparticles were electrodeposited on flexible substrates of metal electrodes, showed a sensitivity of $\mathrm{pH}$ in a wide range of 9 to 5(Santos et al., 2014). And Milne and Connolly (2014) manufactured a disposable $\mathrm{pH}$ sensor by a screen-printing method and an ion selective membrane. It was by the voltage response of a screen-printed $\mathrm{Ag} / \mathrm{AgCl}$ electrode that measured $\mathrm{pH}$ values. To summarize, $\mathrm{pH}$ is also an indispensable index in the microenvironment of wounds and as its changes can act as a significant reference for predicting wound infection and non-healing, more and more sensitive and accurate sensors are developed to monitoring the wound $\mathrm{pH}$ values, which contributes to the promotion of wound healing. 
Moisture level monitoring

Because of the low permeability, skin acts as a barrier to evaporative fluid loss. Fluid loss is expressed as the water vapor transmission rate (WVTR) which is about $8.5 \mathrm{gm}^{-2} \mathrm{~h}^{-1}$ in normal skin(Wu et al., 1996). However, the disruption of skin barrier after injuries often leads to a dramatic increase in WVTR, bringing great loss of electrolytes and fluids. And the WVTRs in ulcers and burns even exhibit tenfold higher than intact skin. The dry environment of wounds not only causes pain, but promotes the progression of necrosis and delays healing. Therefore, moisture level is also a key parameter in wound microenvironment. Hackl et al. (2012) showed that a wet or a moist environment of wounds treated with minced skin micro-grafts, promoted re-epithelialization and decreased contracture. It has also been demonstrated that the wet chamber environment does not damage normal skin, instead, wounds heal faster with less scarring than that in a dry environment(Reish et al., 2009). However, a too much wet wound may lead to maceration. Thus, moisture balance is important, which indicates that if real-time moisture level can be monitored, it will help to take proper measures to promote wound healing.

In the case of wireless moisture sensors, there is a commercialized disposable moisture sensor, WoundSense (Figure 3 ) (McColl et al., 2007; Milne et al., 2016). The hydration map of the wound could be obtained by measuring the impedance of $\mathrm{Ag} / \mathrm{AgCl}$ electrodes inserted into the dressings, which takes advantage of the ionic nature of wound fluid and quantifies fluid volume by using alternating current of varying frequency (McColl et al., 2007). The application of that moisture sensor ensures wound moisture level at an optimal range but also allows less dressing changes(Milne et al., 2016). A new system reported by Messaoud et al. (2018) also rely on electrodes to monitor moisture level. This system includes a wound bed model with a fluid injection system to mimic exudate flow, a flexible sensor array consisting of 14 flexible electrodes and data acquisition software. But for now, it is just used to evaluate the performance of dressing model by putting on the top of them and has not been integrated into dressings. In short, we can see that most devices for moisture monitoring mainly rely on electrodes, and there are few smart dressings that can detect the moisture level of wound microenvironment directly and conveniently.

Uric acid monitoring

Uric acid is the final breakdown product of purine catabolism in our bodies and is in relatively high concentrations within the biofluids(Borderud et al., 2015). Its ubiquity and easy oxidation make it ideal as a biomarker. It is believed that bacteria such as S. aureus and P. aeruginosa. metabolize uric acid rapidly via uricase synthesis, suggesting a rapid decrease in uric acid concentration may be a generic indicator of infection(Dargaville et al., 2013). And according to research of Fernandez et al. (2012), uric acid concentration is also correlated positively with ulcer severity, as chronic venous leg ulcers with no clinical sign of infection were found with elevated uric acid concentration. Sharp et al. (2008) used a carbon fiber sensor laminate and square wave voltammetry to detect the oxidation of urate at $+0.23 \mathrm{~V}$ as a sharp peak free from interference from other metabolites, which achieved optimized responses, and greater sensitivity and specificity.

Protease monitoring

Protease is also one potential biomarker that could predict wound status as proteases are found elevated in non-healing wounds compared to normal or acute healing wounds. A POC test material, WOUNDCHECK from Systagenix, detects elevated total protease activity to assess whether neutrophil associated proteases are present in the wound or not(Serena, 2014). Actually, individual proteases may have varying roles at different times of healing process. Matrix metalloproteinases (MMPs) with the ability to break down all components of the extracellular matrix (ECM) such as collagens and elastin, are the principal proteases involved(McCarty and Percival, 2013). MMPs are produced by fibroblasts that also release tissue inhibitor metalloproteinases (TIMPs) in order to regulate the effects of MMPs(Shah et al., 2012). Higher levels of proteases may be associated with less healing, while higher levels of protease inhibitors may as well(Westby et al., 2020). Thus, there may be a place for the ratio of protease to inhibitor as a predictor of healing. It was reported that the MMP-9/TIMP ratio, and the levels of MMPs and elastase prove to be good prognosticators 
of wound behavior(Snyder et al., 2011). The lower level of MMP and MMP-9/TIMP ratio, the greater chance of improved wound healing. There was one method of label-free, electrochemical impedance immune-sensing developed for the detection and quantification of MMP-9, as well as other two infection biomarkers(Ciani et al., 2012). The detections were performed with gold screen-printed electrodes modified with a specific thiolated antibody, demonstrating the possibility rapid wound infection detection directly from a clinically relevant specimen.

Other proteases such as $\beta$-glucuronidase secreted by Escherichia coli strains are also detected to discover infection(Sadat Ebrahimi et al., 2015). A stable signal for the bacteria presence was sensitively detectable by self-reporting material which was produced by covalently attaching chromogenic and fluorogenic substrates, respectively 4-nitrophenyl- $\beta$-D-glucuronide and 4-methylumbelliferyl- $\beta$-D-glucuronide, to chitosan-based hydrogel. What's more, human neutrophil elastase (HNE) and cathepsin G (CatG) that involved in the pathogenesis of inflammatory disorders, also have the potential to be used as markers for early detection of infection(Hasmann et al., 2011). In a study about sensor materials for detecting HNE and CatG via hydrolysis of the chromogenic substrates, the fluid of infected wounds showed significant higher substrate hydrolysis compared with that of non-infected wounds(Hasmann et al., 2011). But these approaches have not be developed into devices which are able to detect elevated enzyme activities before manifestation of infection directly on bandages. Some studies also detected Lysozymes (Lys) whose altered activity is also significantly connected with wounds colonized with bacteria by using hydrogels that comprise agarose and peptidoglycan components and degrade in the presence of Lys, though not so sufficiently sensitive(Hasmann et al., 2011; Schiffer et al., 2015). In the study of Schneider et al. (2012), the agarose and peptidoglycan layers were modified by covalently attaching polyphenol ferulic acid onto the former and polyethylene glycol-modified laccase onto the latter, then the digestion of peptidoglycan by lysozyme led to the release of laccase that oxidized polyphenols and finally resulted in clearly visible color changes. One study also reported a hydrogel-based dressing for the detection of different types of enzymes, such as serine protease $\alpha$-chymotrypsin, where a modified chitosan hydrogel was functionalized with a fluorogenic substrate which is released by enzymatic degradation(Sadat Ebrahimi and Schönherr, 2014). In a conclusion, protease is also one reliable biomarker for the detection of infection. And interestingly, it seems that the detection of specific pathogenic bacteria can be targeted by using corresponding fluorogenic or chromogenic substrates of to detect different kinds of enzymes.

Nitric oxide monitoring

Nitric oxide (NO) plays a central role in wound healing as a critical molecular signal and mediator(Elliott et al., 2017). It is generated by three isozymes including inducible (iNOS), endothelial (eNOS), and neuronal nitric oxide synthase (nNOS). The two latter, nNOS and eNOS, are constitutively expressed and physiologically catalyze low level NO generation in vascular endothelial cells and neurons, respectively. While, iNOS is inducible only in response to acute inflammatory stimuli, such as invading pathogens and proinflammatory cytokines (Förstermann and Sessa, 2012). And it also has been shown to regulate cytokines that initiate inflammation with downstream effects on the recruitment of keratinocytes(Kobayashi, 2010). More importantly, NO exerts antimicrobial effects at wound site by a combination of nitroastion and oxidation, including direct modification of membrane proteins, lipid peroxidation, and DNA cleavage(Mannick, 2006).

NO deficiency has been established as an important mechanism responsible for poor healing in diabetic foot ulcer (DFU)(Walton et al., 2019). Insufficient NO impedes activities of wound healing cells, such as fibroblasts, macrophages and keratinocytes, which diminishes pathogen clearance, decreases collagen content and strength, and inhibits reepithelization. And studies also found that infection reduced after NO therapy, as well as a less severe immune response, and improved collagen deposition and strength(Shekhter et al., 2019). One study detected NO, uric acid and pyocyanin (PYO) by carbon ultra-microelectrode arrays (CUAs) fabricated on flexible substrates to respectively reflected the actual situation of healing, immune responses, and the presence of P. aeruginosa(Simoska et al., 2020). In addition, as the major metabolic pathway for $\mathrm{NO}$ is conversion to nitrate [NO3-] and nitrite $\left[\mathrm{NO}_{2}-\right]$, collectively termed NOx that are stable metabolites in tissue(Ghasemi and Zahediasl, 2011), many researches chose nitrate as measures of wound NO bioactiv- 
ity(Kapil et al., 2020). Nitrite and nitrate were measured in wound fluid harvested by sponges and in wound cell culture supernatants. However, there have not seen smart dressing that can directly detect NOx in wound fluids. All in all, levels of NO can serve as reliable predictors of wound healing outcomes and more researches should be invested for its use in smart dressings.

Bacteria monitoring

The tissue bacterial level is also one of biological markers that are anticipatory of wound healing. Bacterial colonization will deprive oxygen and nutrients that are necessary for healing, prolong the inflammation period, and eventually make the wound difficult to heal(Shah et al., 2012). Pathogen concentration levels above or equal to $10^{5}$ Colony Forming Units (CFU) per gram are capable of interfering with the wound healing process(Siddiqui and Bernstein, 2010). what's more, if bacteria keep proliferating and encase themselves within extracellular matrix substances of polysaccharides and lipids, biofilm will form which brings resistance to immunological responses and causes infected chronic wounds harder to heal. Therefore, portable biosensors that can screen bacteria quickly will help in wound care.

Thet et al. (2016) developed a prototype wound dressing where the fluorescent dye containing vesicles were mixed with agarose and dispersed within the hydrogel matrix. The dressing switched on a fluorescent color when in contact with pathogenic wound biofilms produced by apathogenic strain (Figure 4 ), which helps identify and treat infected wounds in time. In the study of Currie et al. (2020), a highly sensitive electrospun nanofibrous polyurethane wound dressing was presented, which incorporates a chromogenic probe with a labile ester linkage that can be enzymatically cleaved by bacterial lipase, resulting a rapid chromogenic response. To conclude, bacteria responsive color-changing wound dressings really offer a valuable platform for continuous monitoring of the wound bed facilitating early detection of bacterial infections.

In addition, many of the by-products of bacterial metabolism have characteristic odors, which is often the result of necrosis or extremely poor vascularization of tissues, bacterial colonization or fungal infection in the wound(Stephen-Haynes and Callaghan, 2015). Hence, ideally, the wound odor may be used as an early indicator for the presence pathogens. A new technology, electronic nose (e-nose) embedded with a chemical sensor array, received a widespread application in bacteria detection with advantages of short response time, fast detection speed, low cost and convenience (Yusuf et al., 2013). I,n a proof-of-concept study, enose system was proved to be capable of differentiating methicillin-sensitive Staphylococcus aureus (MSSA), methicillin-resistant Staphylococcus aureus (MRSA), Pseudomonas aeruginosa and others with an accuracy of $78 \%$ within minutes(Saviauk et al., 2018). Another study also reported an electronic nose, Aetholab, able to discriminate between infected versus non-infected wounds a sensitivity of $91 \%$ and a specificity of $71 \%$ with clinical judgment as reference standard(Haalboom et al., 2019). However, there may be 30 gas sensors in an e-nose, leading to higher cross-sensitivity of gas sensors. Liang et al. (2017) proposed to suppress the interference of the sampling stage by removing the information correlated with baseline samples, which turns out significantly effective. However, this new method of detection, e-nose, ideal for the identification of wound infection, has just been used on wound swabs and seems have not been integrated into any smart dressings.

\section{Intelligent on-demand treatment system}

Usually, there are three main methods for the treatment of skin wounds, namely wound dressings, artificial skin substitutes and wound intervention agents. However, as the actual situation of wound healing process is hard to know, it is always in a black-box state and only relies on practical experience of staff to judge. In that way, the wound cannot be implemented with proper measures in time or tailored therapies according to different types and causes of wound, making it difficult to achieve satisfactory results. So those dressings that can be respond to some certain parameters and then offer on-demand treatments such as drug or other therapies, are needed and welcomed in the bright future.

Temperature-sensitive drug release treatment

It is in response to the changes of some parameters in the wound microenvironment that the drug delivery 
mechanism of most smart dressings is. So far, temperature response mechanism seems account for the majority. For example, Lin et al. (2016) reported a thermo-sensitive hydrogel dressing which mainly consists of temperature sensors, LED lights and drug-delivery system. It releases drugs according to changes of wound temperature and gives signals by LED light when the drug is in low density. Besides, the flexible components of the electronics can deform to stretch with skin surface when the hydrogel is stretched, as shown in the Figure 5, and the rigid components with robust interfaces between electronic components and hydrogel matrix, can keep undeformed. That two characteristics make the dressing have a certain degree of flexibility and maintain a stable structure at the same time. However, drug releasing in some dressings rely on a temperature-sensitive hydrogel which is attached to a flexible heater, rather than wound temperature. For example, there was a dermal patch which delivers controlled drug by electronically adjusting the temperature of the hydrogel layer by a flexible heater, where thermo-responsive drug microcarriers are encapsulated(Bagherifard et al., 2016). Similarly, the drug releasing system of one smart flexible wound dressing includes a hydrogel loaded with thermo-responsive drug carriers which releases drugs with the help of an electronically controlled flexible heater for on-demand treatment (Mostafalu et al., 2018). This flexible smart wound dressing may have the potential to significantly impact the treatment of chronic wounds in the near future.

pH-sensitive drug release treatment

$\mathrm{pH}$ is also another vital parameter that changed during wound healing process. It is admitted that stimulisensitive and biodegradable hydrogels are promising biomaterials and excellent candidates for biomedical applications in the controlled drug delivery system of dressings. Zhu et al. (2018) chose peptide-based bis-acrylate with acrylic acid (AAc) to construct hybrid hydrogels which are endowed with pH-dependent swelling property. Drugs are preloaded in hydrogels with interconnected and porous structure, and released when triggered by $\mathrm{pH}$ variation. Recently, there reported a promising $\mathrm{pH}$-responsive wound dressing, whose targeted and controlled delivery of minocycline were realized by halloysite nanotube (HNT) composites modified with different concentration of both poly (lactic-co-glycolic acid) (PLGA) and chitosan(Mohebali and Abdouss, 2020). That pH-responsive release profile of drugs proved effective on the inhibition of grampositive and gram-negative bacteria, making it also be considered as a promising candidate for $\mathrm{pH}$-responsive drug delivery in the treatment of chronic wounds. Similarly, Kiaee et al. (2018) developed a wound dressing that enables active topical drug delivery in response to electrically induced $\mathrm{pH}$ change. The dressing is mainly comprised of a $\mathrm{pH}$ sensitive hydrogel which contains drug loaded chitosan nanoparticles (ChPs), a microfabricated anode coated with a hydrogel layer, and a cathode. When needed, a DC voltage will be applied between the electrodes, which results in a local change in $\mathrm{pH}$ between the electrodes and then drugs are released from ChPs. To sum up, we could see that most $\mathrm{pH}$-sensitive drug release systems integrated in smart dressings rely on stimuli-sensitive hydrogels to trigger drug releasing by the change of $\mathrm{pH}$ values.

\section{Bacteria-responsive drug release treatment}

Besides, there are also some wound dressings relying on the mechanism of bacteria response. For example, a wound dressing which is composed of biocompatible ultraviolet-photocrosslinkable methacrylated gelatin (GelMA) encapsulating both antimicrobial and fluorescent vesicles, has been developed(Zhou, et al., 2018). In the presence of pathogenic bacteria, fluorescent vesicle bilayer membrane will be lysed by toxins secreted by them and then the dressing will show a simple color change as to provide infection warning. In a similar way, antimicrobials will be released from vesicles to inhibit infection. Abdali et al. (2019) also designed a bacteriaresponsive nanofibrous wound dressing where the model biocide, benzyl dimethyl tetradecyl ammonium chloride (BTAC), was incorporated into bacteria-degradable polymer. Because of bacterial activity (both lipase secretion and acidic $\mathrm{pH}$ ), the degradation of polymers was facilitated and caused the release of BTAC. So, that dressing has potential to release antibacterial agents to prevent wound infections without delaying wound healing.

UV/NIR-responsive Wound healing treatment

Besides above, some of drug releasing are triggered by ultraviolet (UV) or near-infrared (NIR) light. For 
example, considering drug resistance and side effects caused by the overuse of antibiotics, Pang et al. (2017) made a wound dressing with UV-responsive antibacterial property, realizing the controllable release of antibiotics only when needed. In their study, levofloxacin (LF) was conjugated to amine-terminated poly (ethylene glycol) through a photolabile linker to obtain a photo-cleavable polyprodrug (LHP), which was then loaded into a poly (vinyl alcohol)/sodium alginate (PVA/SA) wound dressing. The antibiotic LF could be cleaved from LHP under the UV irradiation at $365 \mathrm{~nm}$ and released gradually with an increase in UV irradiation time, which truly realizes the controllable release of antibiotics and avoids the side effect of antibiotic overuse. In their another study, the mechanism behind the smart flexible electronics-integrated wound dressing is same. The main components of that dressing include a temperature sensor, UV light-emitting diodes, and a UV-responsive antibacterial hydrogel. If wound temperature continuously remained higher than a preset threshold value, UV-LEDs will be turned on to warn infection and then UV-responsive hydrogel will release antibiotics in situ to inhibit infection(Pang et al., 2020).

Some studies also exploit NIR to function. For instance, researchers developed a multifunctional platform (TG-NO-B) which was able to selectively bind to Gram-negative bacterial cells and their biofilm matrix on wounds(Zhao et al., 2020). Upon single NIR laser irradiation, TG-NO-B could generate hyperthermia and simultaneously release NO, which would disrupt cell membrane, cause damage and leakage of intracellular components, and finally induce bacteria death. And a smart black phosphorus (BP)-based gel which could function as a biomimetic "skin" to temporarily shield the wound was also reported(Ouyang et al., 2020). It functions as a drug "reservoir" as well, which can generate heat to accelerate blood flow under NIR laser at $808 \mathrm{~nm}$ irradiation, release lidocaine hydrochloride to relief pain. What's more, nanoparticles are also used in NIR triggered dressings. Unlike antibiotic-dependent antibacterial hydrogel, Feng et al. (2021) designed a hydrogel with near-infrared (NIR)-triggered tunable adhesion and bacterial eradication. As the nanoparticles in it has conversion capacity from NIR light into heat, the temperature-sensitive hydrogel can adhere to the wound with its self-deformation property, and achieve an on-demand dressing refreshing. This hydrogel is also proved the capability of bacterial eradication and good treatment effects on wound healing. Sun et al. (2019) utilized up-conversion nanoparticles (UCNPs) to developed a nanocomposite membrane which has antibacterial effects by synergistic photothermal photodynamic therapy. With the irradiation of NIR light at $980 \mathrm{~nm}$ for about five minutes, the nanocomposite membrane could simultaneously generate reactive oxygen species (ROS) as photodynamic therapy, and cause temperature rising as photothermal therapy to restrain infections. Qiao et al. (2020) developed further. They manufactured a smart hydrogel-based wound dressing (Figure 6 ) capable of monitoring bacterial infection via a $\mathrm{pH}$-responsive fluorescence and providing on-demand treatment. The hydrogels are composed of four parts: polyvinyl alcohol (PVA), UCNPs, silica nanoparticles modified with $\mathrm{Cy} 3$ and $\mathrm{Cy} 5$ (SNP-Cy3/Cy5), and gentamicin sulfate-UV cleavable linkerPEG methoxyl (GS-Linker-MPEG). Once infection detected, the hydrogels will be irradiated with NIR light, UCNP are able to convert NIR to UV light to trigger the release of drug, gentamicin, from the hydrogels for antibacterial treatment. Similarly, Huang et al. (2021) constructed a complex film through the formation of hydrogen bonds and hydrophobic interactions between hydroxypropyl methylcellulose (HPMC) and epigallocatechin-3-gallate (EGCG). The incorporation of $\mathrm{CuS}$ nanoparticles into the EGCG/HPMC complex film allowed it to trigger EGCG release under near-infrared (NIR) exposure. And EGCG could also be released by tuning $\mathrm{pH}$ or temperature of the release medium. When bacteria grow, $\mathrm{pH}$ rises. On the one hand, it caused the transformation of EGCG, which leads to the color change of the complex films as infection indication. On the other hand, it helps EGCG generate $\mathrm{H} 2 \mathrm{O} 2$ to kill bacteria. To conclude, these smart materials and dressings are expected to provide a new strategy for self-reporting and effective on-demand treatment of wound infection in the near future.

\section{Oxygen therapy}

Researchers have demonstrated that delayed healing in diabetic chronic wounds is a result of damaged neovascularization in response to hypoxia(Botusan et al., 2008; Thangarajah et al., 2010). It was believed that the sole source of oxygen available for biochemical processes of wound healing was derived from the systemic circulation. But now it is supported that skin can utilize oxygen from the atmosphere. Therefore, hyperbaric oxygen therapy (HBOT)(Andrade and Santos, 2016) and topical oxygen therapy (TOT)(Roe 
et al., 2010), employing gaseous oxygen delivery, can reduce hypoxia and may be an effective way for the treatment of diabetic wounds. The problem is both of them perform poorly in penetrating the skin. In this front, smart dressings seem more efficient in local delivery of dissolved oxygen. For instance, Chen et al. (2020) developed a patch-like wound dressing which fills with gel beads containing active Synechococcus elongatus (S. elongatus) PCC7942. In this patch, oxygen is generated by PCC7942, so a local moist HBO atmosphere for dissolved oxygen delivery can be established. Besides, chlamydomonas reinhardtii cultured in a bioartificial scaffolds, has also been used to produce oxygen for tissue repair(Hopfner et al., 2014).

Oxygen therapy can not only promote tissue recovery, but also inhibit infection. For example, photodynamic inactivation (PDI) is one of ways to eradicate the bacterial infection and overcome bacterial resistance. It involves the combination of nontoxic dyes with visible light to produce ROS in a photochemical reaction between the excited state of the photosensitizer and ambient oxygen. Sun et al. (2020) reported a biocompatible $\gamma$-PGA-based nanofibers matrix doped with photosensitizer TMPyP for anti-infection therapy. Different from traditional antibacterial materials that exert bactericidal activities by releasing bactericides, this nanofibrous mat itself is not bactericidal, but can provide potent bactericidal activity upon visible light irradiation by releasing the cytotoxic ROS. There is a novel wound dressing based on oxygen-generating materials (sodium percarbonate/calcium peroxide) that delivered oxygen through microchannels. The in vivo tests demonstrated rapid wound healing by using these oxygen-generating materials(Lo et al., 2013; Chandra et al., 2015). Chen et al. (2017) created a smart bandage which can simultaneously monitor and inhibit wound infection. The main components of this bandage are luminescent porous silicon (LuPSi) particles loadecd with ciprofloxacin (CIP). In infected wounds, the ROS can cause the oxidation of LuPSi, then triggers the release of CIP molecules to inhibit bacterial activity. Chlorophyll is also a natural photosensitizer can produce ROS combined with laser irradiation(Gunes et al., 2017). Another study designed a bioactive living hydrogel using spirulina platensis (SP) which contains abundant chlorophyll for hypoxia elimination and bacteria killing(Li et al., 2020). The ROS production of SP gel promoted by $650 \mathrm{~nm}$ laser irradiation could significantly inhibit the infection of both gram-positive S. aureus and gram-negative E. coli, and it was proven to be effective for both in vitro cellular hypoxia and in vivo tissue hypoxia elimination.

\section{Conclusion and Future prospects}

As we have previously reviewed, parameters such as temperature, $\mathrm{pH}$, hydration, in the wound microenvironment can not only affect the healing process but also tell the actual status of wound, making them helpful indicators for noninvasive monitoring and early detection of non-healing wounds or even infection. All the researches mentioned in this review demonstrated that more and more focus on developing techniques and innovation, and designing much more practical smart dressings with more accurate and sensitive sensors to measure $\mathrm{pH}$, temperature, moisture level, uric acid, protease, nitric oxide, microbial load and etc. automatically and continuously. Those successful experiments in vitro models make the clinical application of smart dressings promising for wound repair. However, existed smart dressings encompass just one or two aforementioned parameters, instead of simultaneously measuring as many of them as possible. In addition, combining on-demand treatment systems with sensors would be necessary for a so called ideal smart dressing, which allows in-time therapy be gave to the deteriorative wound if data measured by sensors indicates worsening situation of wound healing. As mentioned above, we know that there are many kinds of mechanism for on-demand treatment, such as drug release therapy with temperature or $\mathrm{pH}$ sensitive, bacteria or UV/NIR responsive, and oxygen therapy. But existed smart dressings (Table 1 ) with both functions of monitoring and treatment are relatively rare, and need to be invested more resource and research to develop, and further performed to validate their feasibility in vivo experiments or even in clinical. What's more, due to the complexity of wound regeneration, the fluctuation of various parameters and the corresponding biological behavior of the wound need to be further matched. In this case, the indication that a certain wound status or exact situation of healing process concluded from one change in one parameter will be more reliable.

To sum up, the full automation of real-time monitoring, infection warning and self-controllable drug release or other therapy in one dressing is hoped to be realized in the not far future, to be applicated in acute wounds, diabetic wounds, ulcer and the so on. This new-type skin bioelectronic system with the function 
of wound monitoring and on-demand treatment is definitely a great trend for wound repair, and is believed to promote wound healing, relieve patients' pain, save amounts of money for their families and reduce the burden of social medical care.

\section{Author contributions}

YFZ investigated and summarized the literature, and wrote the original draft. YW, YYH, XFW, QQF, and ZCW conducted deep review and editing. WYZ, TWK, YNX, YQL and LM helped revise the paper and gave some advice. XZW, DL and WQT supervised and applied for funds. All authors have read and approved this manuscript for publication.

\section{Acknowledgements}

This work was supported by grants from National Natural Science Foundation of China (No. 81671918), National Key Research Program of China (No. 2016YFC1101004) and Zhejiang Provincial Medical and Healthy Science Foundation of China (No. 2018KY874).

\section{Compliance with ethics guidelines}

The authors declare that there is no conflict of interest. This study does not contain any studies with human or animal subjects performed by any of the authors.

\section{References}

Abdali Z, Logsetty S, Liu S. Bacteria-Responsive Single and Core-Shell Nanofibrous Membranes Based on Polycaprolactone/Poly(ethylene succinate) for On-Demand Release of Biocides. ACS Omega. 2019. 4(2): 4063-4070. doi: 10.1021/acsomega.8b03137

Amendola S, Lodato R, Manzari S, Occhiuzzi C, Marrocco G. RFID Technology for IoT-Based PersonalHealthcare in Smart Spaces. IEEE INTERNET OF THINGS JOURNAL. 2014. 1(2): 144-152. doi: 10.1109/JIOT.2014.2313981

Andrade SM, Santos IC. Hyperbaric oxygen therapy for wound care. Rev Gaucha Enferm. 2016. 37(2): e59257. doi: 10.1590/1983-1447.2016.02.59257

Arafa AA, Nada AA, Ibrahim AY, Sajkiewicz P, Zahran MK, Hakeim OA. Preparation and characterization of smart therapeutic pH-sensitive wound dressing from red cabbage extract and chitosan hydrogel. Int J Biol Macromol. 2021. 182: 1820-1831. doi: 10.1016/j.ijbiomac.2021.05.167

Armstrong DG, Lipsky BA, Polis AB, Abramson MA. Does dermal thermometry predict clinical outcome in diabetic foot infection? Analysis of data from the SIDESTEP* trial. Int Wound J. 2006. 3(4): 302-7. doi: 10.1111/j.1742-481X.2006.00269.x

Bagherifard S, Tamayol A, Mostafalu P, et al. Dermal Patch with Integrated Flexible Heater for on Demand Drug Delivery. Adv Healthc Mater. 2016. 5(1): 175-84. doi: 10.1002/adhm.201500357

Bjarnsholt T. The role of bacterial biofilms in chronic infections. APMIS Suppl. 2013. (136): 1-51. doi: 10.1111/apm.12099

Borderud SP, Li Y, Burkhalter JE, Sheffer CE and Ostroff JS. Electronic cigarette use among patients with cancer: Characteristics of electronic cigarette users and their smoking cessation outcomes. Cancer. doi: 10.1002/cncr.28811. Cancer. 2015. 121(5): 800. doi: 10.1002/ cncr.28811

Botusan IR, Sunkari VG, Savu O, et al. Stabilization of HIF-1alpha is critical to improve wound healing in diabetic mice. Proc Natl Acad Sci U S A. 2008. 105(49): 19426-31. doi: 10.1073/pnas.0805230105

Broussard KC, Powers JG. Wound dressings: selecting the most appropriate type. Am J Clin Dermatol. 2013. 14(6): 449-59. 
Chalitangkoon J, Monvisade P. Synthesis of chitosan-based polymeric dyes as colorimetric pH-sensing materials: Potential for food and biomedical applications. Carbohydr Polym. 2021. 260: 117836. doi: $10.1007 / \mathrm{s} 40257-013-0046-4$

Chandra PK, Ross CL, Smith LC, et al. Peroxide-based oxygen generating topical wound dressing for enhancing healing of dermal wounds. Wound Repair Regen. 2015. 23(6): 830-41. doi: 10.1111/wrr.12324

Chen H, Cheng Y, Tian J, et al. Dissolved oxygen from microalgae-gel patch promotes chronic wound healing in diabetes. Sci Adv. 2020. 6(20): eaba4311. doi: 10.1126/sciadv.aba4311

Chen X, Wo F, Jin Y, Tan J, Lai Y, Wu J. Drug-Porous Silicon Dual Luminescent System for Monitoring and Inhibition of Wound Infection. ACS Nano. 2017. 11(8): 7938-7949. doi: 10.1021/acsnano.7b02471

Ciani I, Schulze H, Corrigan DK, et al. Development of immunosensors for direct detection of three wound infection biomarkers at point of care using electrochemical impedance spectroscopy. Biosens Bioelectron. 2012. 31(1): 413-8. doi: 10.1016/j.bios.2011.11.004

Currie S, Shariatzadeh FJ, Singh H, Logsetty S, Liu S. Highly Sensitive Bacteria-Responsive Membranes Consisting of Core-Shell Polyurethane Polyvinylpyrrolidone Electrospun Nanofibers for In Situ Detection of Bacterial Infections. ACS Appl Mater Interfaces. 2020. 12(41): 45859-45872. doi: 10.1021/acsami.0c14213

Dargaville TR, Farrugia BL, Broadbent JA, Pace S, Upton Z, Voelcker NH. Sensors and imaging for wound healing: a review. Biosens Bioelectron. 2013. 41: 30-42. doi: 10.1016/j.bios.2012.09.029

Dini V, Salvo P, Janowska A, Di Francesco F, Barbini A, Romanelli M. Correlation Between Wound Temperature Obtained With an Infrared Camera and Clinical Wound Bed Score in Venous Leg Ulcers. Wounds. 2015. 27(10): 274-8.

Elliott J, Duay J, Simoska O, Shear JB, Stevenson KJ. Gold Nanoparticle Modified Transparent Carbon Ultramicroelectrode Arrays for the Selective and Sensitive Electroanalytical Detection of Nitric Oxide. Anal Chem. 2017. 89(2): 1267-1274. doi: 10.1021/acs.analchem.6b03987

Feng L, Shi W, Chen Q, et al. Smart Asymmetric Hydrogel with Integrated Multi-Functions of NIR-Triggered Tunable Adhesion, Self-Deformation, and Bacterial Eradication. Adv Healthc Mater. 2021: e2100784. doi: 10.1002/adhm.202100784

Fernandez ML, Upton Z, Edwards H, Finlayson K, Shooter GK. Elevated uric acid correlates with wound severity. Int Wound J. 2012. 9(2): 139-49. doi: 10.1111/j.1742-481X.2011.00870.x

Fierheller M, Sibbald RG. A clinical investigation into the relationship between increased periwound skin temperature and local wound infection in patients with chronic leg ulcers. Adv Skin Wound Care. 2010. 23(8): 369-79; quiz 380-1. doi: 10.1097/01.ASW.0000383197.28192.98

Förstermann U, Sessa WC. Nitric oxide synthases: regulation and function. Eur Heart J. 2012. 33(7): 829-37, 837a-837d. doi: 10.1093/eurheartj/ehr304

Ghasemi A, Zahediasl S. Is nitric oxide a hormone. Iran Biomed J. 2011. 15(3): 59-65.

Gunes S, Tamburaci S, Dalay MC, Deliloglu Gurhan I. In vitro evaluation of Spirulina platensis extract incorporated skin cream with its wound healing and antioxidant activities. Pharm Biol. 2017. 55(1): 18241832. doi: $10.1080 / 13880209.2017 .1331249$

Haalboom M, Gerritsen JW, van der Palen J. Differentiation between infected and non-infected wounds using an electronic nose. Clin Microbiol Infect. 2019. 25(10): 1288.e1-1288.e6. doi: 10.1016/j.cmi.2019.03.018

Hackl F, Bergmann J, Granter SR, et al. Epidermal regeneration by micrograft transplantation with immediate 100-fold expansion. Plast Reconstr Surg. 2012. 129(3): 443e-452e. doi: 10.1097/PRS.0b013e318241289c 
Hasmann A, Gewessler U, Hulla E, et al. Sensor materials for the detection of human neutrophil elastase and cathepsin G activity in wound fluid. Exp Dermatol. 2011. 20(6): 508-13. doi: 10.1111/j.16000625.2011.01256.x

Hasmann A, Wehrschuetz-Sigl E, Kanzler G, et al. Novel peptidoglycan-based diagnostic devices for detection of wound infection. Diagn Microbiol Infect Dis. 2011. 71(1): 12-23. doi: 10.1016/j.diagmicrobio.2010.09.009

Hattori Y, Falgout L, Lee W, et al. Multifunctional skin-like electronics for quantitative, clinical monitoring of cutaneous wound healing. Adv Healthc Mater. 2014. 3(10): 1597-607. doi: 10.1002/adhm.201400073

Hazenberg CE, van Netten JJ, van Baal SG, Bus SA. Assessment of signs of foot infection in diabetes patients using photographic foot imaging and infrared thermography. Diabetes Technol Ther. 2014. 16(6): 370-7. doi: 10.1089/dia.2013.0251

Hopfner U, Schenck TL, Chávez MN, et al. Development of photosynthetic biomaterials for in vitro tissue engineering. Acta Biomater. 2014. 10(6): 2712-7. doi: 10.1016/j.actbio.2013.12.055

Huang TW, Lu HT, Ho YC, Lu KY, Wang P, Mi FL. A smart and active film with tunable drug release and color change abilities for detection and inhibition of bacterial growth. Mater Sci Eng C Mater Biol Appl. 2021. 118: 111396. doi: 10.1016/j.msec.2020.111396

Jankowska DA, Bannwarth MB, Schulenburg C, et al. Simultaneous detection of pH value and glucose concentrations for wound monitoring applications. Biosens Bioelectron. 2017. 87: 312-319. doi: 10.1016/j.bios.2016.08.072

Jones EM, Cochrane CA, Percival SL. The Effect of $\mathrm{pH}$ on the Extracellular Matrix and Biofilms. Adv Wound Care (New Rochelle). 2015. 4(7): 431-439. doi: 10.1089/wound.2014.0538

Kapil V, Khambata RS, Jones DA, et al. The Noncanonical Pathway for In Vivo Nitric Oxide Generation: The Nitrate-Nitrite-Nitric Oxide Pathway. Pharmacol Rev. 2020. 72(3): 692-766. doi: 10.1124/pr.120.019240

Kassal P, Zubak M, Scheipl G, Mohr GJ, Steinberg MD, Steinberg IM. Smart bandage with wireless connectivity for optical monitoring of pH. Sensors and Actuators B. 2017. 246. doi: 10.1016/j.snb.2017.02.095

Kiaee G, Mostafalu P, Samandari M, Sonkusale S. A pH-Mediated Electronic Wound Dressing for Controlled Drug Delivery. Adv Healthc Mater. 2018. 7(18): e1800396. doi: 10.1002/adhm.201800396

Kobayashi Y. The regulatory role of nitric oxide in proinflammatory cytokine expression during the induction and resolution of inflammation. J Leukoc Biol. 2010. 88(6): 1157-62. doi: 10.1189/jlb.0310149

Kruse CR, Nuutila K, Lee CC, et al. The external microenvironment of healing skin wounds. Wound Repair Regen. 2015. 23(4): 456-64. doi: 10.1111/wrr.12303

Lee TY, Kim KB, Han SK, Jeong SH, Dhong ES. Skin Hydration Level as a Predictor for Diabetic Wound Healing: A Retrospective Study. Plast Reconstr Surg. 2019. 143(4): 848e-856e. doi: 10.1097/PRS.0000000000005474

Lee W, Kwon O, Lee DS, Yeo WH. Fabrication and Characterization of a Conformal Skin-like Electronic System for Quantitative, Cutaneous Wound Management. J Vis Exp. 2015. (103). doi: 10.3791/53037

Li W, Wang S, Zhong D, Du Z, Zhou M. A Bioactive Living Hydrogel: Photosynthetic Bacteria Mediated Hypoxia Elimination and Bacteriakgilling to Promote Infected Wound Healing. 2020. 4(1). doi:10.1002/adtp.202000107

Liang Z, Tian F, Zhang C, Sun H, Liu X, Yang SX. A correlated information removing based interference suppression technique in electronic nose for detection of bacteria. Anal Chim Acta. 2017. 986: 145-152. doi: 10.1016/j.aca.2017.07.028

Lin S, Yuk H, Zhang T, et al. Stretchable Hydrogel Electronics and Devices. Adv Mater. 2016. 28(22): 4497-505. doi: 10.1002/adma.201504152 
Lindley LE, Stojadinovic O, Pastar I, Tomic-Canic M. Biology and Biomarkers for Wound Healing. Plast Reconstr Surg. 2016. 138(3 Suppl): 18S-28S. doi: 10.1097/PRS.0000000000002682

Liu L, Li X, Nagao M, Elias AL, Narain R, Chung HJ. A pH-Indicating Colorimetric Tough Hydrogel Patch towards Applications in a Substrate for Smart Wound Dressings. Polymers (Basel). 2017. 9(11). doi: 10.3390/polym9110558

Lo JF, Brennan M, Merchant Z, et al. Microfluidic wound bandage: localized oxygen modulation of collagen maturation. Wound Repair Regen. 2013. 21(2): 226-34. doi: 10.1111/wrr.12021

Lou D, Pang Q, Pei X, et al. Flexible wound healing system for pro-regeneration, temperature monitoring and infection early warning. Biosens Bioelectron. 2020. 162: 112275. doi: 10.1016/j.bios.2020.112275

Mannick JB. Immunoregulatory and antimicrobial effects of nitrogen oxides. Proc Am Thorac Soc. 2006. 3(2): 161-5. doi: 10.1513/pats.200505-048BG

Martinez-Jimenez MA, Aguilar-Garcia J, Valdes-Rodriguez R, et al. Local use of insulin in wounds of diabetic patients: higher temperature, fibrosis, and angiogenesis. Plast Reconstr Surg. 2013. 132(6): 1015e-1019e. doi: 10.1097/PRS.0b013e3182a806f0

Matzeua G, Losaccob M, Parduccib E, et al. Skin temperature monitoring by a wireless sensor. 2011 IEEE. 2011 : 3533-3535.

Maver T, Gradišnik L, Smrke DM, Stana Kleinschek K, Maver U. Systematic Evaluation of a DiclofenacLoaded Carboxymethyl Cellulose-Based Wound Dressing and Its Release Performance with Changing pH and Temperature. AAPS PharmSciTech. 2019. 20(1): 29. doi: 10.1208/s12249-018-1236-4

McCarty SM, Percival SL. Proteases and Delayed Wound Healing. Adv Wound Care (New Rochelle). 2013. 2(8): 438-447. doi: 10.1089/wound.2012.0370

McColl D, Cartlidge B, Connolly P. Real-time monitoring of moisture levels in wound dressings in vitro: an experimental study. Int J Surg. 2007. 5(5): 316-22. doi: 10.1016/j.ijsu.2007.02.008

Menke NB, Ward KR, Witten TM, Bonchev DG, Diegelmann RF. Impaired wound healing. Clin Dermatol. 2007. 25(1): 19-25. doi: 10.1016/j.clindermatol.2006.12.005

Messaoud M, Marsiquet C, Revol-Cavalier F, Rat V, Marchand G. Flexible sensors for real-time monitoring of moisture levels in wound dressings. J Wound Care. 2018. 27(6): 385-391. doi: 10.12968/jowc.2018.27.6.385

Milici S, Amendola S, Bianco A, Marrocco G. Epidermal RFID passive sensor for body temperature measurements. 2014 .

Milne SD, Connolly P. The influence of different dressings on the $\mathrm{pH}$ of the wound environment. J Wound Care. 2014. 23(2): 53-4, 56-7. doi: 10.12968/jowc.2014.23.2.53

Milne SD, Seoudi I, Al Hamad H, et al. A wearable wound moisture sensor as an indicator for wound dressing change: an observational study of wound moisture and status. Int Wound J. 2016. 13(6): 1309-1314. doi: 10.1111/iwj.12521

Mohebali A, Abdouss M. Layered biocompatible pH-responsive antibacterial composite film based on $\mathrm{HNT} / \mathrm{PLGA} /$ chitosan for controlled release of minocycline as burn wound dressing. Int J Biol Macromol. 2020. 164: 4193-4204. doi: 10.1016/j.ijbiomac.2020.09.004

Mostafalu P, Tamayol A, Rahimi R, et al. Smart Bandage for Monitoring and Treatment of Chronic Wounds. Small. 2018 : e1703509. doi: 10.1002/smll.201703509

Moura LI, Dias AM, Carvalho E, de Sousa HC. Recent advances on the development of wound dressings for diabetic foot ulcer treatment-a review. Acta Biomater. 2013. 9(7): 7093-114. doi: 10.1016/j.actbio.2013.03.033 
Nakagami G, Sanada H, lizaka S, et al. Predicting delayed pressure ulcer healing using thermography: a prospective cohort study. J Wound Care. 2010. 19(11): 465-6, 468, 470 passim. doi: 10.12968/jowc.2010.19.11.79695

Occhiuzzi, C., Caizzone, S., Marrocco, G. Passive UHF RFID antennas for sensing applications: Principles, methods, and classifcations. Antennas \& Propagation Magazine. 2013. 55(6): 14-34. doi: 10.1109/MAP.2013.6781700

Ouyang J, Ji X, Zhang X, et al. In situ sprayed NIR-responsive, analgesic black phosphorus-based gel for diabetic ulcer treatment. Proc Natl Acad Sci U S A. 2020. 117(46): 28667-28677. doi: 10.1073/pnas.2016268117

Pang Q, Lou D, Li S, et al. Smart Flexible Electronics-Integrated Wound Dressing for Real-Time Monitoring and On-Demand Treatment of Infected Wounds. Adv Sci (Weinh). 2020. 7(6): 1902673. doi: 10.1002/advs.201902673

Pang Q, Zheng X, Luo Y, Ma L, Gao C. A photo-cleavable polyprodrug-loaded wound dressing with UVresponsive antibacterial property. J Mater Chem B. 2017. 5(45): 8975-8982. doi: 10.1039/c7tb01696d

Percival SL, McCarty S, Hunt JA, Woods EJ. The effects of pH on wound healing, biofilms, and antimicrobial efficacy. Wound Repair Regen. 2014. 22(2): 174-86. doi: 10.1111/wrr.12125

Phair J, Newton L, McCormac C, Cardosi MF, Leslie R, Davis J. A disposable sensor for point of care wound pH monitoring. Analyst. 2011. 136(22): 4692-5. doi: 10.1039/c1an15675f

Power G, Moore Z, O'Connor T. Measurement of $\mathrm{pH}$, exudate composition and temperature in wound healing: a systematic review. J Wound Care. 2017. 26(7): 381-397. doi: 10.12968/jowc.2017.26.7.381

Qiao B, Pang Q, Yuan P, Luo Y, Ma L. Smart wound dressing for infection monitoring and NIR-triggered antibacterial treatment. Biomater Sci. 2020. 8(6): 1649-1657. doi: 10.1039/c9bm02060h

Reish RG, Zuhaili B, Bergmann J, et al. Modulation of scarring in a liquid environment in the Yorkshire pig. Wound Repair Regen. 2009. 17(6): 806-16. doi: 10.1111/j.1524-475X.2009.00546.x

Roe DF, Gibbins BL, Ladizinsky DA. Topical dissolved oxygen penetrates skin: model and method. J Surg Res. 2010. 159(1): e29-36. doi: 10.1016/j.jss.2009.10.039

Sadat Ebrahimi MM, Schönherr H. Enzyme-sensing chitosan hydrogels. Langmuir. 2014. 30(26): 7842-50. doi: 10.1021/la501482u

Sadat Ebrahimi MM, Voss Y, Schönherr H. Rapid Detection of Escherichia coli via Enzymatically Triggered Reactions in Self-Reporting Chitosan Hydrogels. ACS Appl Mater Interfaces. 2015. 7(36): 20190-9. doi: 10.1021/acsami.5b05746

Santos L, Neto JP, Crespo A, et al. WO3 nanoparticle-based conformable pH sensor. ACS Appl Mater Interfaces. 2014. 6(15): 12226-34. doi: 10.1021/am501724h

Saviauk T, Kiiski JP, Nieminen MK, et al. Electronic Nose in the Detection of Wound Infection Bacteria from Bacterial Cultures: A Proof-of-Principle Study. Eur Surg Res. 2018. 59(1-2): 1-11. doi: 10.1159/000485461

Schiffer D, Verient V, Luschnig D, et al. Lysozyme-responsive polymer systems for detection of infection. Eng Life Sci. 2015. 15: 368-375. doi: 10.1002/bit.26070

Schneider KP, Gewessler U, Flock T, et al. Signal enhancement in polysaccharide based sensors for infections by incorporation of chemically modified laccase. N Biotechnol. 2012. 29(4): 502-9. doi: 10.1016/j.nbt.2012.03.005

Schneider LA, Korber A, Grabbe S, Dissemond J. Influence of $\mathrm{pH}$ on wound-healing: a new perspective for wound-therapy. Arch Dermatol Res. 2007. 298(9): 413-20. doi: 10.1007/s00403-006-0713-x 
Serena TE. Development of a Novel Technique to Collect Proteases from Chronic Wounds. Adv Wound Care (New Rochelle). 2014. 3(12): 729-732. doi: 10.1089/wound.2013.0463

Shah JM, Omar E, Pai DR, Sood S. Cellular events and biomarkers of wound healing. Indian J Plast Surg. 2012. 45(2): 220-8. doi: 10.4103/0970-0358.101282

Sharp D, Forsythe S, Davis J. Carbon fibre composites: integrated electrochemical sensors for wound management. J Biochem. 2008. 144(1): 87-93. doi: 10.1093/jb/mvn045

Sharpe JR, Booth S, Jubin K, Jordan NR, Lawrence-Watt DJ, Dheansa BS. Progression of wound pH during the course of healing in burns. J Burn Care Res. 2013. 34(3): e201-8. doi: 10.1097/BCR.0b013e31825d5569

Shekhter AB, Pekshev AV, Vagapov AB, et al. Physicochemical parameters of NO-containing gas flow affect wound healing therapy. An experimental study. Eur J Pharm Sci. 2019. 128: 193-201. doi: 10.1016/j.ejps.2018.11.034

Shi L, Ramsay S, Ermis R, Carson D. pH in the bacteria-contaminated wound and its impact on clostridium histolyticum collagenase activity: implications for the use of collagenase wound debridement agents. J Wound Ostomy Continence Nurs. 2011. 38(5): 514-21. doi: 10.1097/WON.0b013e31822ad034

Siah CJ, Childs C. Thermographic mapping of the abdomen in healthy subjects and patients after enterostoma. J Wound Care. 2015. 24(3): 112; 114-20. doi: 10.12968/jowc.2015.24.3.112

Siah CR, Childs C, Chia CK, Cheng K. An observational study of temperature and thermal images of surgical wounds for detecting delayed wound healing within four days after surgery. J Clin Nurs. 2019. 28(11-12): 2285-2295. doi: $10.1111 /$ jocn. 14832

Siddiqui AR, Bernstein JM. Chronic wound infection: facts and controversies. Clin Dermatol. 2010. 28(5): 519-26. doi: 10.1016/j.clindermatol.2010.03.009

Simoska O, Duay J, Stevenson KJ. Electrochemical Detection of Multianalyte Biomarkers in Wound Healing Efficacy. ACS Sens. 2020. 5(11): 3547-3557. doi: 10.1021/acssensors.0c01697

Simões D, Miguel SP, Ribeiro MP, Coutinho P, Mendonça AG, Correia IJ. Recent advances on antimicrobial wound dressing: A review. Eur J Pharm Biopharm. 2018. 127: 130-141. doi: 10.1016/j.ejpb.2018.02.022

Snyder RJ, Driver V, Fife CE, et al. Using a diagnostic tool to identify elevated protease activity levels in chronic and stalled wounds: a consensus panel discussion. Ostomy Wound Manage. 2011. 57(12): 36-46.

Stephen-Haynes J, Callaghan R. Zinc-impregnated and odour-control two-layer compression. Br J Nurs. 2015. 24(15): S52, S54-7. doi: 10.12968/bjon.2015.24.Sup15.S52

Sun BK, Siprashvili Z, Khavari PA. Advances in skin grafting and treatment of cutaneous wounds. Science. 2014. 346(6212): 941-5. doi: 10.1126/science.1253836

Sun J, Song L, Fan Y, et al. Synergistic Photodynamic and Photothermal Antibacterial Nanocomposite Membrane Triggered by Single NIR Light Source. ACS Appl Mater Interfaces. 2019. 11(30): 26581-26589. doi: $10.1021 /$ acsami.9b07037

Sun L, Song L, Zhang X, Zhou R, Yin J, Luan S. Poly( $\gamma$-glutamic acid)-based electrospun nanofibrous mats with photodynamic therapy for effectively combating wound infection. Mater Sci Eng C Mater Biol Appl. 2020. 113: 110936. doi: 10.1016/j.msec.2020.110936

Tamayol A, Akbari M, Zilberman Y, et al. Flexible pH-Sensing Hydrogel Fibers for Epidermal Applications. Adv Healthc Mater. 2016. 5(6): 711-9. doi: 10.1002/adhm.201500553

Thangarajah H, Vial IN, Grogan RH, et al. HIF-1alpha dysfunction in diabetes. Cell Cycle. 2010. 9(1): 75-9. doi: 10.4161/cc.9.1.10371 
Thet NT, Alves DR, Bean JE, et al. Prototype Development of the Intelligent Hydrogel Wound Dressing and Its Efficacy in the Detection of Model Pathogenic Wound Biofilms. ACS Appl Mater Interfaces. 2016. 8(24): 14909-19. doi: 10.1021/acsami.5b07372

Tottoli EM, Dorati R, Genta I, Chiesa E, Pisani S, Conti B. Skin Wound Healing Process and New Emerging Technologies for Skin Wound Care and Regeneration. Pharmaceutics. 2020. 12(8). doi: 10.3390/pharmaceutics 12080735

Ulrich S, Osypova A, Panzarasa G, Rossi RM, Bruns N, Boesel LF. Pyranine-Modified Amphiphilic Polymer Conetworks as Fluorescent Ratiometric pH Sensors. Macromol Rapid Commun. 2019. 40(21): e1900360. doi: $10.1002 /$ marc. 201900360

Walton DM, Minton SD, Cook AD. The potential of transdermal nitric oxide treatment for diabetic peripheral neuropathy and diabetic foot ulcers. Diabetes Metab Syndr. 2019. 13(5): 3053-3056. doi: 10.1016/j.dsx.2018.07.003

Westby MJ, Norman G, Watson R, Cullum NA, Dumville JC. Protease activity as a prognostic factor for wound healing in complex wounds. Wound Repair Regen. 2020. 28(5): 631-644. doi: 10.1111/wrr.12835

Woo KY, Sibbald RG. A cross-sectional validation study of using NERDS and STONEES to assess bacterial burden. Ostomy Wound Manage. 2009. 55(8): 40-8.

Wu P, Nelson EA, Reid WH, Ruckley CV, Gaylor JD. Water vapour transmission rates in burns and chronic leg ulcers: influence of wound dressings and comparison with in vitro evaluation. Biomaterials. 1996. 17(14): 1373-7. doi: 10.1016/0142-9612(96)87277-2

Yusuf N, Omar MI, Zakaria A, Abdullah AA. Diagnosis of bacteria for diabetic foot infection using electronic nose technology. 2013 IEEE Conference on Wireless Sensor. 2013. 114-118: 114-118. doi: 10.1186/s12859015-0601-5

Zeng J, Hu M, Wang W, Chen H, Qin Y. NO2-Sensing Properties of Porous WO3 Gas Sensor Based on Anodized Sputtered Tungsten Thin Film. Sensors Actuators B Chem.. 2012. 161: 447-452.

Zhao B, Wang H, Dong W, et al. A multifunctional platform with single-NIR-laser-triggered photothermal and NO release for synergistic therapy against multidrug-resistant Gram-negative bacteria and their biofilms. J Nanobiotechnology. 2020. 18(1): 59. doi: 10.1186/s12951-020-00614-5

Zhou J, Yao D, Qian Z, et al. Bacteria-responsive intelligent wound dressing: Simultaneous In situ detection and inhibition of bacterial infection for accelerated wound healing. Biomaterials. 2018. 161: 11-23. doi: 10.1016/j.biomaterials.2018.01.024

Zhu J, Han H, Ye TT, et al. Biodegradable and pH Sensitive Peptide Based Hydrogel as Controlled Release System for Antibacterial Wound Dressing Application. Molecules. 2018. 23(12). doi: 10.3390/molecules23123383

\section{Hosted file}

table.docx available at https://authorea.com/users/442094/articles/542447-smart-dressingsfor-wound-monitoring-and-treatment-new-type-skin-bioelectronic-systems-applicated-inwound-repair

\section{Hosted file}

Figures.docx available at https://authorea.com/users/442094/articles/542447-smart-dressingsfor-wound-monitoring-and-treatment-new-type-skin-bioelectronic-systems-applicated-inwound-repair 